\title{
Systematic Measurements of Doppler-Coincidence Spectra for Positron Annihilation in Pure Metals and Semiconductors
}

\author{
W. DenG ${ }^{a}$, D. PliszKa ${ }^{b}$, R.S. Brusa ${ }^{a}$, G.P. KARWASZ ${ }^{a, b}$ \\ AND A. ZECCA ${ }^{a}$ \\ ${ }^{a}$ Istituto Nazionale per la Fisica della Materia, Unita di Trento \\ 38050 Povo, Italy \\ ${ }^{b}$ Institute of Physics, Pomeranian Pedagogical University, 76-200 Słupsk, Poland
}

(Received January 21, 2002; revised version April 4, 2002)

\begin{abstract}
Doppler-broadening measurements of the electron-positron annihilation line in twenty six elements are presented. The adopted coincidence technique allows to reduce the background and point out the contribution of positron annihilation with core electrons. The changes of the high momentum contribution is presented for selected examples and a semiempirical analysis of the dependence on electronic structure is performed. Measured data are in a good agreement with recent theoretical calculations and can be used to identification of impurities surrounding open volume defects.
\end{abstract}

PACS numbers: $71.60 .+\mathrm{z}, 78.70 . \mathrm{Bj}$

\section{Introduction}

Positron annihilation techniques (lifetime, Doppler broadening, angular correlation) present some unique advantages if compared to other solid-state analysis methods. The positron spectroscopy is nondestructive, differently than, for example, secondary-ion mass spectroscopy; it does not modify the defects concentration, in contrast to the Rutherford backward scattering in channeling; it allows the depth 
profiling down to a few micrometers which is not possible directly in X-ray techniques; it does not require special preparation of samples, like an electron scanning or transmission microscopy.

Positrons injected into matter annihilate in a short time (less than $10^{-8} \mathrm{~s}$ ) and all the information on the annihilation site is brought by two (or three) gamma quanta. In particular, the two-dimensional angular correlation of the positron-electron annihilation radiation (ACAR) allows to evidence first-principle aspects of metal monocrystals, like Fermi surfaces [1]. On the other hand, positron lifetimes in pure elements show characteristic values, ranging from 105 for $\mathrm{Ni}$ to $220 \mathrm{ps}$ for $\mathrm{Si}[2]$; rise of the lifetime indicates presence of defects. The lifetimes for defects are quantified, say 300 ps for bi-vacancies in $\mathrm{Si}, 321 \mathrm{ps}$ for tri-vacancies and so on [2]. Such a quantization is not well established for the third technique, i.e. the Doppler broadening of the $511 \mathrm{keV}$ annihilation line. Only recently, it proved possible to quantify experimentally the type of defects in some solids, like Si-monocrystals irradiated with $\mathrm{He}^{+}$ions [3].

The positron defectoscopies, at present, show also some drawbacks: ACAR gives a straightforward information only for monocrystals, the depth profiling with lifetime measurements requires beam-bunching techniques [4], the Doppler broadening examines rather the sample "morphology" and does not give information on the chemical nature of the annihilation site. Improving the chemical selectivity of the most versatile among positron techniques, i.e. Doppler broadening, would open new practical applications at low cost. Only recently first works on improving chemical sensitivity of the Doppler broadening have been done [5-10].

\section{Experimental}

Pure metals and semiconductors were studied with a positron annihilation Doppler technique, modified in order to reduce the spurious backgrounds. In solids positron slows down to thermal energies in less than $10^{-13} \mathrm{~s}$. In the annihilation process, the momentum of the electron-positron pair, $p$, is transferred to the photon pair. The momentum of thermalized positron is negligible compared to electron momentum. Therefore, the difference between the momentum of the photon originating from the annihilation "at rest" and from annihilation of a pair possessing non-zero momentum brings direct information on electron momentum.

The longitudinal, i.e. in the direction of the photon propagation, component $p_{\mathrm{L}}$ of momentum $p$ causes a Doppler shift of the annihilation line of $511 \mathrm{keV}$ energy. This shift $\Delta E$ is related to $p_{\mathrm{L}}$ by an approximate relation $\Delta E=p_{\mathrm{L}} c / 2$. Since numerous annihilation events contribute to the complete Doppler spectrum, the $511 \mathrm{keV}$ line is broadened in both directions. In defect-free metals positrons annihilate preferentially with valence electrons, i.e. with the low momentum which does differ much for different chemical elements. To study the annihilation with core electrons, the background must be reduced using a two-detector coincidence system, as first proposed by Lynn and Goland [5], see Fig. 1. 


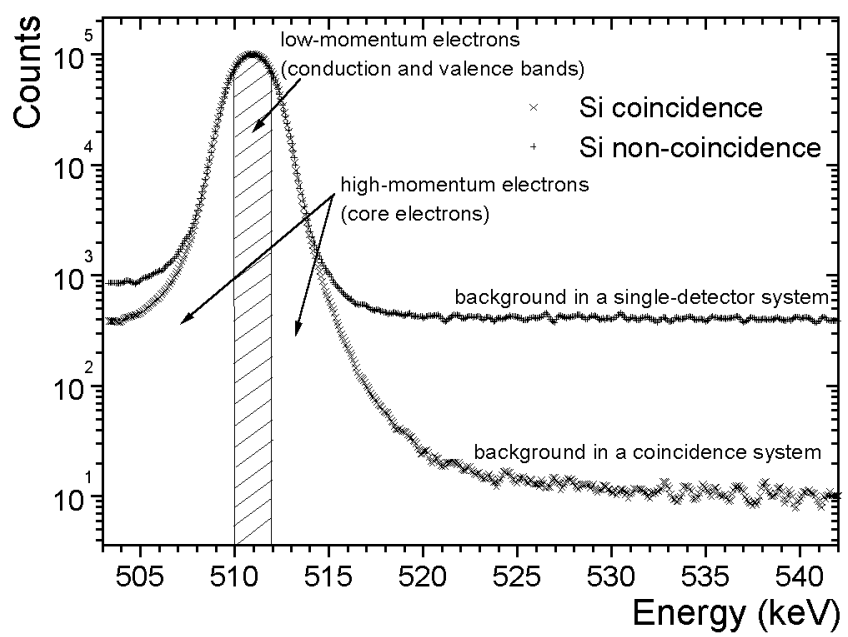

Fig. 1. Typical shape of the typical annihilation line. Let us note lowering of the background level using the coincidence technique.

In Fig. 1 we use the "experimental" abscissa scale - the value of the photon energy as read from detector. In next figures we present a double scale - the "experimental" one and, additionally, with the electron momentum projection $p_{\mathrm{L}}$. The momentum $p_{\mathrm{L}}$ is expressed in $m_{0} c$ units through the relation $p_{\mathrm{L}} / m_{0} c=$ $2 \Delta E / E$, where $c$ is the light velocity and $m_{0}$ - electron mass. In some figures, when trying to correlate the observed momentum to the mean theoretical momenta of electrons on a given atomic shell, we use atomic units instead, with 1 a.u. = $(1 / 137) m_{0} c$ (= momentum of the electron in Bohr's atom).

As the main detector we used a high purity Ge (HPGe) Canberra detector (16\% efficiency) with a resolution of $1.3 \mathrm{keV}$ at $511 \mathrm{keV}$ and with $2 \mu$ s shaping time in the HPGe spectroscopy amplifier. The auxiliary detector supplying the coincidence signal was an $\mathrm{NaI}(\mathrm{Tl})$ scintillator. Detectors were placed in collinear geometry with the Ge detector at a distance of $23 \mathrm{~cm}$ from the sample. Detectors were shielded with lead in order to decrease the noise. With this set up, we get a coincidence count rate of about 140 counts/s in the $511 \mathrm{keV}$ peak and a peak to background ratio of $1.5 \times 10^{4}$ on the high energy side of the peak. A rise of the distance between detectors improves the coincidence detection but lowers the counting rate. The coincidence spectra were taken until more than $1 \times 10^{7}$ counts had accumulated in the peak. After every spectra was recorded, the energy scale of the system was calibrated by taking the peak position of the $356.23 \mathrm{keV}$ gamma line supplied by a small ${ }^{133} \mathrm{Ba}$ source in the single detector mode. In these spectra, each channel was $77.6 \mathrm{eV}$ energy wide.

As a secondary technique we used positron lifetime measurements. This technique was applied in order to check if the samples were defect-free. In a lifetime 
spectrometer the $1.28 \mathrm{MeV}$ de-excitation photon emitted from ${ }^{22} \mathrm{Na}$ radioactive source in about $3 \mathrm{ps}$ after the positron emission is used as a start signal and the $511 \mathrm{keV}$ annihilation photon as the stop signal. The measurements have been carried out with a fast-fast coincidence system [11] with Hamamatsu R2083Q photomultipliers and $\mathrm{BaF}_{2}$ scintillators. The resolution of the apparatus was $160 \mathrm{ps}$ (FWHM). More than $1 \times 10^{6}$ counts were accumulated in every lifetime spectrum.

Both for lifetime and Doppler broadening measurements a ${ }^{22} \mathrm{Na}$ positron source was sandwiched between two similar samples. The ${ }^{22} \mathrm{Na}$ positron sources (10 $\mu \mathrm{Ci}$ for lifetime measurements and $15 \mu \mathrm{Ci}$ for Doppler-broadening measurements) prepared by deposition of ${ }^{22} \mathrm{NaCl}$ were sandwiched between two kapton foils $7 \mu \mathrm{m}$ thick.

\section{Sample preparation}

All samples were obtained from Goodfellow, UK. Silicon (100) monocrystals were obtained from SGS, Italy. In as-received samples several types of traps for positrons can exist: open volume defects from vacancies to vacancy clusters, dislocations. For this reason measurements were done both on as-received and annealed samples, and Doppler curves and lifetimes compared. Most of the samples were annealed for three hours at about $5 \times 10^{-7} \mathrm{mbar}$ (annealing temperatures are given in Table, column three) using a vacuum oven. Some of the samples $(\mathrm{Cr}, \mathrm{Co}$, $\mathrm{Au}$ ) were annealed in two cycles of three hours. Mo, Hf, and $\mathrm{W}$ were annealed at higher temperature in vacuum by electron bombardment. The samples were left to cool down from the annealing temperature to room temperature in the oven by turning off the heating. Normally this procedure took more than twelve hours.

\section{Data analysis}

\subsection{Lifetime}

The lifetime spectra were analyzed by PATFIT packet. The source component (382 ps) varies from $7 \%$ for B to $22 \%$ for Au. For defect-free materials only one component is present in the spectra and for these samples we give the characteristic free positron annihilation lifetime in the bulk of the system $\tau_{\mathrm{b}}$, see Table. For samples containing defects we have characterized our lifetime spectrum by the average lifetime $\tau_{\mathrm{m}}$ with an error of $1 \mathrm{ps}$. In the case of a pure element without defects $\tau_{\mathrm{m}}$ equals $\tau_{\mathrm{b}}$. If open volume defects are present, $\tau_{\mathrm{m}}$ is larger than $\tau_{\mathrm{b}}$. For selected elements ( $\mathrm{Mo}, \mathrm{Ag}, \mathrm{Pb}, \mathrm{Au}$ ) we present single components of the lifetime spectra before and after annealing. 
TABLE

Measured lifetimes for selected elements. Atomic number $Z$, temperature used for annealing, a verage positron lifetime (error $\pm 1 \mathrm{ps}$ ). First, second and the intensity of the second lifetime component for elements whose average lifetime differs from the bulk lifetime. For elements presented in Fig. 10 (Mo, Ag, Pb, Au) we present both lifetimes before annealing (lower rows) and after final annealing (upper rows). In the last column we present theoretical bulk lifetime from [17].

\begin{tabular}{|c|c|c|c|c|c|c|c|c|}
\hline$Z$ & $\begin{array}{l}\text { Ele- } \\
\text { ment }^{a}\end{array}$ & Purity & $\begin{array}{c}\text { Temperature } \\
{\left[{ }^{\circ} \mathrm{C}\right]^{b}}\end{array}$ & $\begin{array}{c}\tau_{\mathrm{m}} \\
{[\mathrm{ps}]}\end{array}$ & $\begin{array}{c}\tau_{1}^{c} \\
{[\mathrm{ps}]}\end{array}$ & $\begin{array}{c}\tau_{2} \\
{[\mathrm{ps}]}\end{array}$ & $\begin{array}{c}I_{2}^{d} \\
{[\%]}\end{array}$ & $\begin{array}{c}\text { Theory } \\
{[17]}\end{array}$ \\
\hline 13 & $\mathrm{Al}$ & - & 500 & 160 & - & - & - & 166 \\
\hline 14 & $\mathrm{Si}$ & - & - & 220 & - & - & - & 221 \\
\hline 22 & $\mathrm{Ti}$ & 99.9 & 1100 & 145 & - & - & - & 146 \\
\hline 23 & $\mathrm{~V}$ & 99.7 & 1100 & 123 & - & - & - & 116 \\
\hline 24 & $\mathrm{Cr}$ & 99.5 & 1100 & 157 & 83 & $248 \pm 2$ & 44.8 & 101 \\
\hline 25 & Mn & 99.5 & - & 150 & 121 & $279 \pm 6$ & 17.7 & 103 \\
\hline 26 & $\mathrm{Fe}$ & 99.99 & 1000 & 123 & 110.7 & $257 \pm 10$ & 8.6 & 101 \\
\hline 27 & $\mathrm{Co}$ & 99.95 & 1100 & 117 & 99.6 & $245 \pm 10$ & 11.7 & 97 \\
\hline 28 & $\mathrm{Ni}$ & 99.98 & 1000 & 105 & - & - & - & 96 \\
\hline 29 & $\mathrm{Cu}$ & 99.8 & 850 & 115 & - & - & - & 106 \\
\hline 30 & $\mathrm{Zn}$ & 99.9 & 250 & 149 & - & - & - & 134 \\
\hline 31 & $\mathrm{Ga}$ & 99.9 & 237 & & - & - & - & - \\
\hline 40 & $\mathrm{Zr}$ & 99.8 & 1100 & 159 & - & - & - & 159 \\
\hline \multirow[t]{2}{*}{42} & Mo & 99.9 & $>1500$ & 119 & - & - & - & 111 \\
\hline & & & & 123 & 117.4 & $660 \pm 60$ & 1.0 & \\
\hline \multirow[t]{2}{*}{47} & $\mathrm{Ag}$ & 99.9 & 850 & 135 & - & - & - & 120 \\
\hline & & & & 188 & 122.7 & $240 \pm 2$ & 57.0 & \\
\hline 74 & W & 99.8 & $>2000$ & 115 & 103 & $245 \pm 15$ & 8.6 & 100 \\
\hline 78 & $\mathrm{Pt}$ & 99.5 & 1000 & 167 & 142 & $282 \pm 8$ & 18.0 & 94 \\
\hline \multirow[t]{2}{*}{79} & $\mathrm{Au}$ & 99.99 & 850 & 123 & 119 & $230 \pm 56$ & 3.7 & 107 \\
\hline & & & & 190 & 181 & $290 \pm 40$ & 7.9 & \\
\hline \multirow[t]{2}{*}{82} & $\mathrm{~Pb}$ & 99.5 & 250 & 206 & - & - & - & 187 \\
\hline & & & & 210 & 210.3 & $960 \pm 75$ & 1.2 & \\
\hline
\end{tabular}

${ }^{a}$ Elements in bold are single crystals: Al (111), Si (100), Ni (111), Cu (100), Zn (00), Mo (100).

${ }^{b}$ Temperature of annealing - in bold samples with more than one annealing step.

${ }^{c}$ Errors on $\tau_{1}$ are typically $\pm 1 \mathrm{ps}$, apart from $\mathrm{Au}( \pm 2 \mathrm{ps})$.

${ }^{d}$ Errors on $I_{2}$ are $\pm 1 \%$. 


\subsection{Doppler coincidence}

Variations of the Doppler-broadening shape due to the core-electron momentum distribution in different elements are shown in Fig. 2. Only the right-hand side of the peak shown in Fig. 1 is used in our analysis. The low-energy part of the spectra like in Fig. 1 contains contributions from the three-photon decay of positronium; this part is also influenced by the effect of the incomplete charge collection in the Ge detector. The high-energy part is influenced only by the background (cosmic-ray and ambient stray radiation) contribution. The use of the coincidence technique lowers the background at large Doppler shifts - the curves are narrower than without coincidence, see Fig. 1. This allows to evidence contributions from electrons with large momentum values.

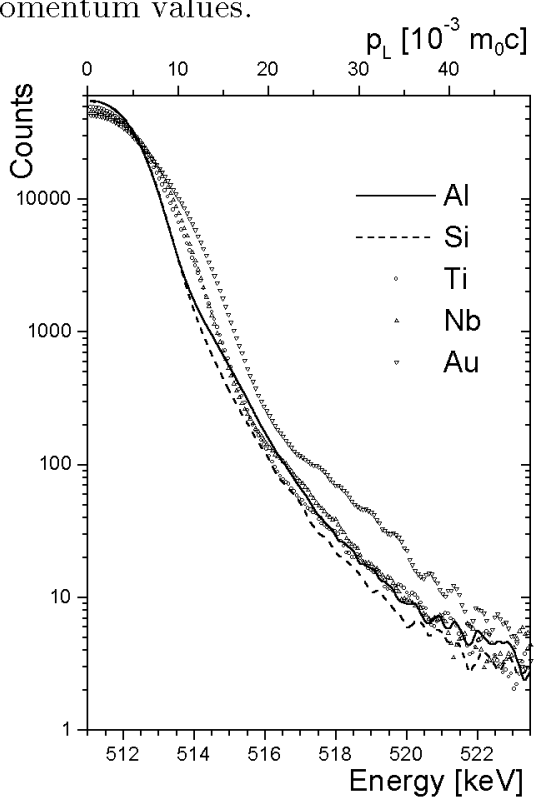

Fig. 2. High-energy part of the annihilation line for Al, Ti, Nb, Au and Si. 9-point, movable smoothing and normalization of the total number of counts were done on these spectra.

The change in shape of the Doppler broadened spectrum due to annihilation with core electrons is very small. To emphasize the differences between the different elements we have followed the idea introduced in [12] by constructing ratio curves, i.e. every spectrum is divided by a spectrum of a material chosen as reference. The choice of the reference material is straightforward in studies of doping of pure materials. For example Kauppinen et al. [7] used pure CdTe as reference curves to evidence vacancies due to $\mathrm{In}, \mathrm{Cl}$ and I (iodium).

In the case of pure materials, the choice is not so clear. For example Alatalo et al. [8] used $\mathrm{Si}$ to evidence defects in $\mathrm{Fe}, \mathrm{Cu}$ and differences between diamond 
and graphite. Szpala et al. [9] used defect-free Si to evidence differences between $\mathrm{Ni}, \mathrm{Cu}, \mathrm{Sb}$ and Ge. Baranowski et al. [10] used Ni sample as the reference for their studies of pure metals and alloys. A general-purpose choice would be to use an element with low contribution from high-momenta electrons, i.e. an element with low atomic number $Z$. On the other hand, a reference standard should be easy-to-handle and, also, to be calculated by the theory. For the sake of theory, Ghosh et al. [13] used $\mathrm{Al}$ as the reference. But we note that even some recent calculations in $\mathrm{Al}$ [14] disagree by a factor of about 20 at zero-momentum although agree within the experimental error bar for momenta higher than $20 m_{0} c$.

From Fig. 2 we see that the Al curves lie above the Si-one for all $p_{\mathrm{L}}$ values higher than $10 m_{0} c$. It means that the contribution from high-momenta electrons is lower in $\mathrm{Si}$ than in $\mathrm{Al}$, in spite of the fact that $\mathrm{Si}$ contains one $3 p$ electron more than Al. This is, qualitatively, probably due to a "looser" lattice structure in Si (lattice constant $a=5.43 \AA)$ compared to $\mathrm{Al}(a=4.05 \AA)$, reflected also in a longer positron lifetime, see Table.

A constant background calculated as the average of the counts in one hundred channels from 607 to $615 \mathrm{keV}$ was subtracted in every spectrum. Then a source correction was applied. It was obtained by measurements on two thick kapton samples formed by compacting several kapton foils, each $125 \mu \mathrm{m}$ thick. The Doppler spectrum of kapton was subtracted from all measured spectra with a percentage dependent on the sample and equal to that used in the evaluation of the average lifetime reported in Table. Without this correction errors up to $-15 \%$ in the height of the peaks of the ratio curves can be made in the heavier elements. Also the relative height of the peaks, where more than one peak is present, change. Finally a smoothing, spline routine on 9 points was applied to the corrected data and the spectra were normalized to a total area of $1 \times 10^{6}$ from 511 to $530 \mathrm{keV}$ $\left(p_{\mathrm{L}}\right.$ from 0 to $\left.74.3 \times 10^{-3} m_{0} c\right)$. These corrected and normalized spectra were used to make the ratio with a reference spectrum of a suitable element.

To minimize the noise on the ratio curves, particular care was taken to prepare the reference curve. The reference curve was obtained from Czochralski-type, p-doped (100) silicon, with $5 \Omega \mathrm{cm}$ resistivity and $5 \times 10^{-17} \mathrm{~cm}^{3}$ oxygen contents. This curve has been smoothed by a moving 9 -point spline averaging in the range from $511 \mathrm{keV}$ to $518 \mathrm{keV}$; above $518 \mathrm{keV}$ it has been first smoothed and then approximated by an exponential formula.

The analytical fit procedure reduces the noise in the high-momentum part of spectrum for the reference sample. On the other hand, the 9-point averaging in principle does not reduce the casual spread of data in the "measured" sample. Therefore, because of low counting rate at high momenta, we do not show the ratio curves above $50 \times 10^{-3} m_{0} c$ - where the expected experimental error in the ratio is of the same order of magnitude as the signal.

Ratio curves for the same elements (we have chosen $\mathrm{Ni}$ and $\mathrm{Au}$ ) obtained by using different reference ( $\mathrm{Al}$ or $\mathrm{Si}$ ) are shown in Fig. 3. Both reference elements 


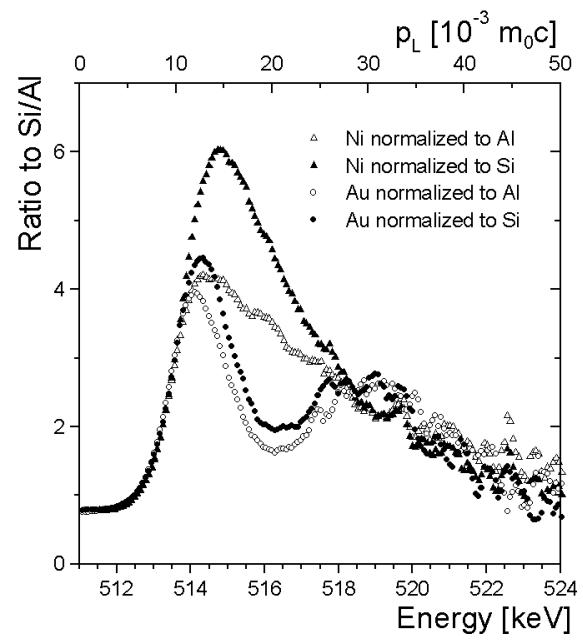

Fig. 3. Comparison of ratio curves using as a reference element $\mathrm{Al}$ and Si.

give similar curves; however the maxima are higher in the case of $\mathrm{Si}$, for reasons explained above. As a consequence, also position of the maxima in the ratio curves depends on the choice of the reference material, see Fig. 3.

\section{Results}

\subsection{Lifetime data}

Lifetime values for pure metals were measured in numerous works $[15,16]$, therefore we do not perform a detailed analysis and in Table we present the measured values only for elements which are significant for further discussion. Generally, our lifetime data agree well with acknowledged theoretical values $[2,17]$. In particular in Al and Si our values (155 and $220 \mathrm{ps)} \mathrm{coincide} \mathrm{with} \mathrm{the} \mathrm{experimental}$ uncertainty with the recent theory [2]. In $\mathrm{Cr}$ and Fe we have some more serious discrepancy: the intensities of the second lifetime component are about $10 \%$ indicating that not all defects have been removed and/or some impurities are present. As far as in Cr the annealing process has reduced the second component intensity by a factor of 4 , in Fe the second component remained almost the same after annealing. We hypothesize some impurities for the latter metal. In Ga (melting temperature $29.8^{\circ} \mathrm{C}$ ) some thermal vacancies are for sure present - our measured lifetime is $237 \mathrm{ps}$, closer to $260 \mathrm{ps}$ measured in a liquid phase [18] than to $190 \mathrm{ps}$ reported for the solid phase [16].

\subsection{Coincidence spectra}

In figures from 4 to 8 we present Si-related curves for 26 elements presently studied. It can be seen especially well for fourth-period metals (Figs. 5, 6) that the height of the peaks in the ratio curves rises with the rise of the number of 
$d$-electrons. However, this rising order is not perfect. For Cr the peak should be probably higher in a non-defected sample (our lifetime analysis shows presence of $40 \%$ of a long component). On the other hand, Mn and Fe show a "correct" order, although both of them are defected. The highest peak is seen in (111)-oriented defect-free $\mathrm{Ni}$ monocrystal (with eight $d$-electrons). Next elements in the 4 th period, with ten $d$ electrons, $\mathrm{Cu}\left(4 s^{1} 3 d^{10}\right)$ and $\mathrm{Zn}\left(4 s^{2} 3 d^{10}\right)$ show somewhat similar, broad curves; the peak height in $\mathrm{Cu}$ is the same as in $\mathrm{Ni}$ but in $\mathrm{Zn}$ much lower.

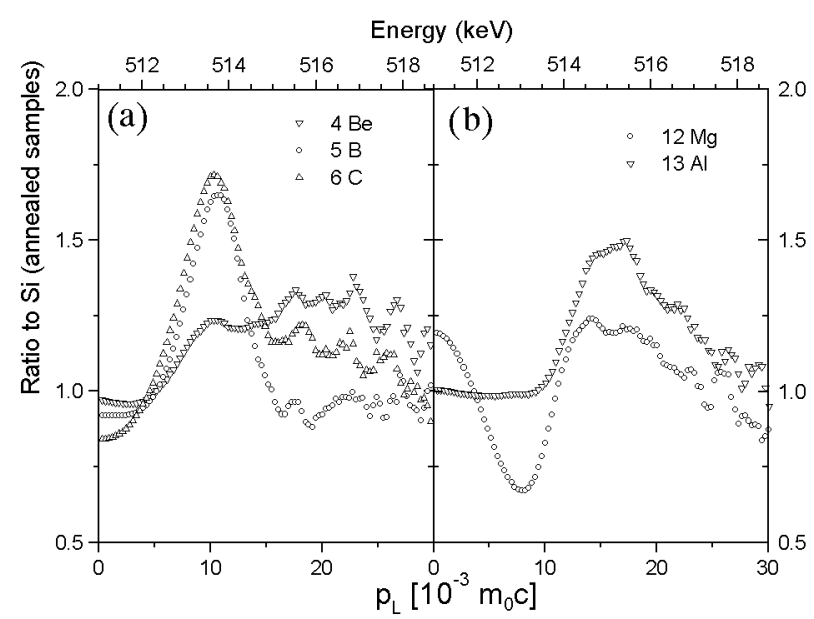

Fig. 4. Doppler broadening coincidence ratio curves (ratio to Si) for specific Mendeleyev-period elements ((a) Be, B, C - second-period elements; (b) Mg, Al third-period elements).

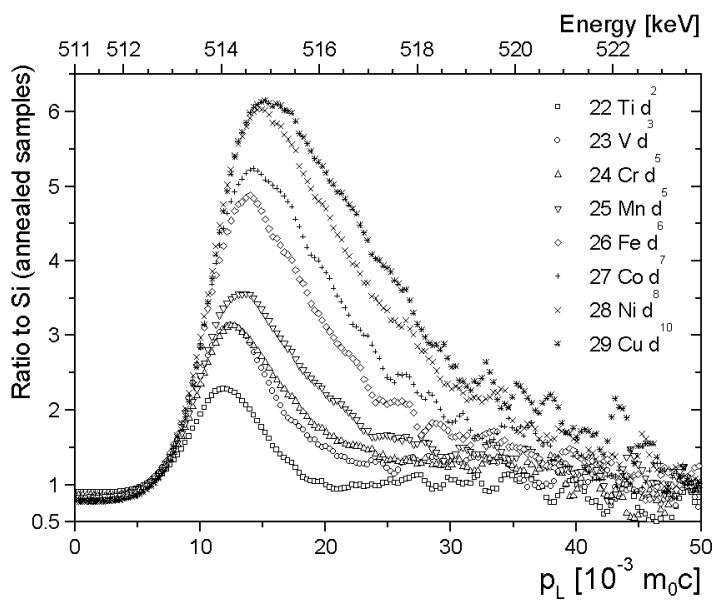

Fig. 5. Doppler broadening coincidence ratio curves (ratio to $\mathrm{Si}$ ) for specific Mendeleyev-period elements ( $\mathrm{Ti}, \mathrm{V}, \mathrm{Cr}, \mathrm{Mn}, \mathrm{Fe}, \mathrm{Co}, \mathrm{Ni}, \mathrm{Cu}$ - fourth-period elements). 


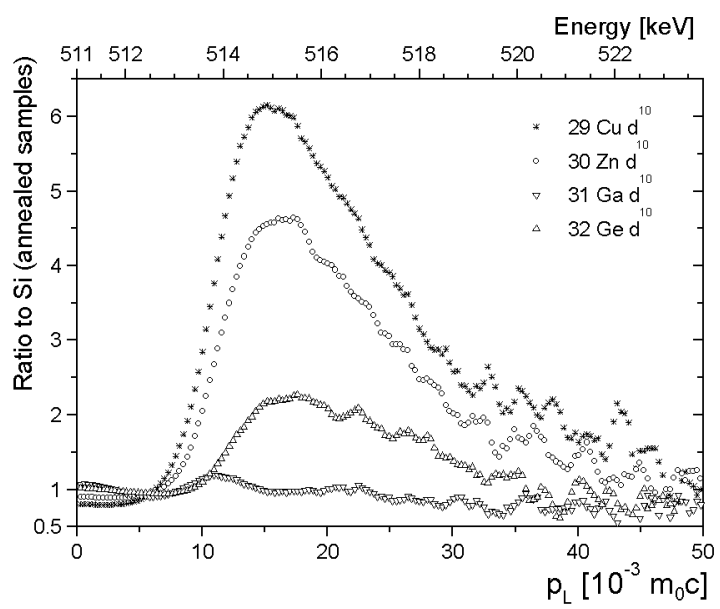

Fig. 6. Doppler broadening coincidence ratio curves (ratio to $\mathrm{Si}$ ) for specific Mendeleyev-period elements ( $\mathrm{Cu}, \mathrm{Zn}, \mathrm{Ga}, \mathrm{Ge}$ - fourth-period elements).

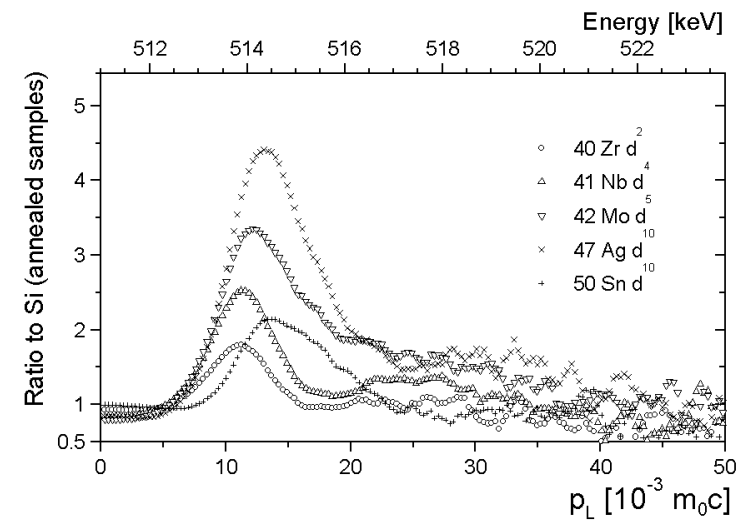

Fig. 7. Doppler broadening coincidence ratio curves (ratio to $\mathrm{Si}$ ) for specific Mendeleyev-period elements ( $\mathrm{Zr}, \mathrm{Nb}, \mathrm{Mo}, \mathrm{Ag}$, Sn - fifth-period elements).

A long-range aim of the present measurements would be to settle characteristic features of spectra for each element. It seems from Fig. 4 to Fig. 8 that each chemical element can be well distinguished thanks to the position, amplitude and shape of the peaks in the ratio curves. However, exact predictions of the ratio curves require complex, first-principle theoretical analysis [8, 13] taking into account electronic mean momenta and the probabilities of positron annihilation with electrons of separate orbitals - the overlap of the wave function of the annihilating positron with electronic wave functions and shielding effects of inner electrons by "outermost" ones. Therefore, below we try some qualitative-like analysis, only.

The amplitudes of peaks seem to reflect the growing annihilation with high-momentum, i.e. $d$-electrons. Therefore, one expects that the peaks rise with the filling-up of the $d$-orbitals for the elements belonging to the same period of the 


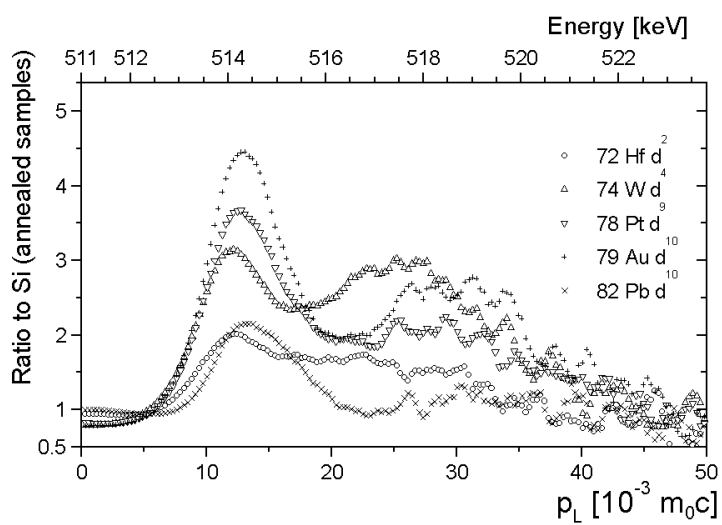

Fig. 8. Doppler broadening coincidence ratio curves (ratio to $\mathrm{Si}$ ) for specific Mendeleyev-period elements (Hf, W, Pt, Au, Pb - sixth-period elements).

Mendeleyev table. This feature is observed in the main picture resulting from our Figs. 4-8, compare for example the $\mathrm{Mn}\left(3 d^{5}\right)$ and $\mathrm{Fe}\left(3 d^{6}\right)$ pair in Figs. 5, 6 or Hf $\left(5 d^{2}\right), \mathrm{W}\left(5 d^{4}\right), \mathrm{Pt}\left(5 d^{9}\right)$, Au $\left(5 d^{10}\right)$ series in Fig. 8.

On the other hand, the height of the peak reflects also the probability of the annihilation with the "outermost" electrons. This seems to be the case of $\mathrm{Zn}$ with the maximum of the ratio curve slightly lower than $\mathrm{Cu}$ : the $\mathrm{Zn}$ atom has the $4 s$ orbital, the "outermost" one, filled completely, which causes the probability of annihilation with $3 d$ electrons to be lower than in $\mathrm{Cu}$ (with only one $4 s$ electron). A similar behavior can be noticed for $5^{\circ}$ and $6^{\circ}$ period metals: the peak for $\mathrm{Ag}$ $\left(5 s^{1}\right)$ is higher than the peak for $\operatorname{Sn}\left(5 s^{2} p^{2}\right)$; the peak for Au $\left(6 s^{1}\right)$ is higher than the peak for $\mathrm{Pb}\left(6 s^{2} p^{2}\right)$, see Figs. 7 and 8 , respectively.

Comparing selected elements belonging to the same groups of the period table, like the VI group (Cr, Mo, W), see Fig. 9a, one notes an almost the same shape of the (first) peak and a growing "shoulder" in Mo, passing to another
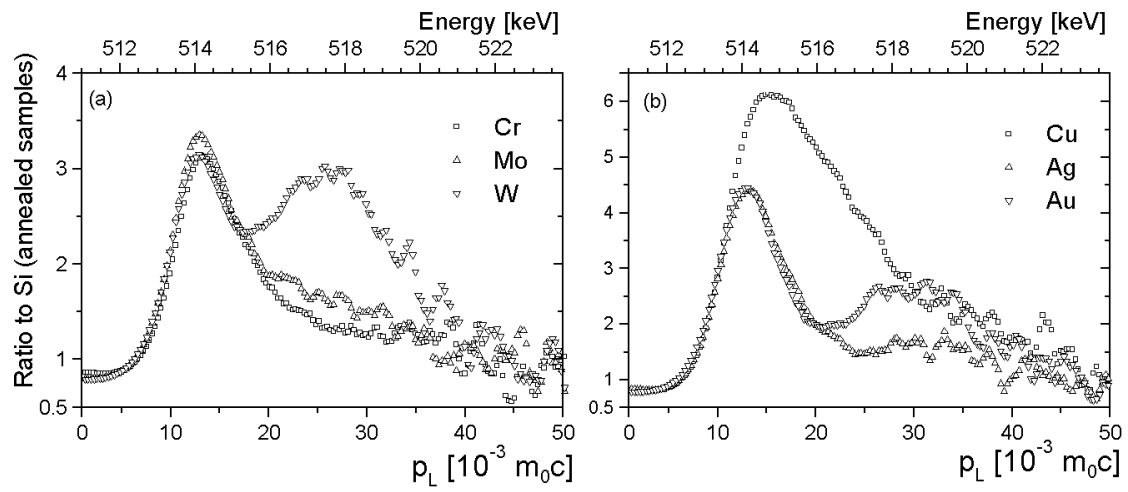

Fig. 9. Comparison of ratio curves Doppler broadening spectra for the: (a) Cr, Mo W, (b) $\mathrm{Cu}, \mathrm{Ag}, \mathrm{Au}$. 
distinct peak for $W$. Somewhat similar behavior can be observed for the I-b group $(\mathrm{Cu}, \mathrm{Ag}, \mathrm{Au})$ : the first peak in $\mathrm{Au}$ and $\mathrm{Ag}$ is identical and $\mathrm{Au}$ develops a second peak at about $30 \times 10^{-3} m_{0} c$ (Fig. $9 \mathrm{~b}$ ). Copper shows a very broad curve like, on the other hand, predicted by the theory [13].

We note that the ratio curves show a strong dependence on presence of positron traps, see Fig. 10. In this figure we compare samples of $\mathrm{Ag}, \mathrm{Au}$, Mo and $\mathrm{Pb}$ as-received and after annealing. For all cases the ratio curves rise in their maxima after annealing, with the biggest difference in the case of $\mathrm{Ag}$ (rise by $20 \%$ of the first peak) and $\mathrm{Au}$ (rise in both peaks by almost $50 \%$ ). We note that such a rise is accompanied by the fall of the mean lifetime, see Table.
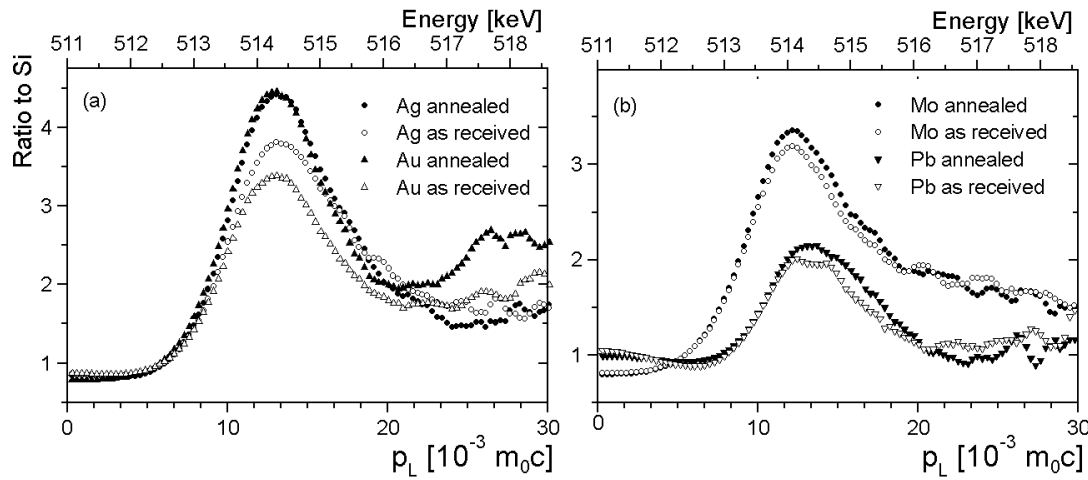

Fig. 10. Ratio of the Doppler broadening spectra with Si spectrum before and after annealing for (a) Ag, Au, (b) Mo, $\mathrm{Pb}$.

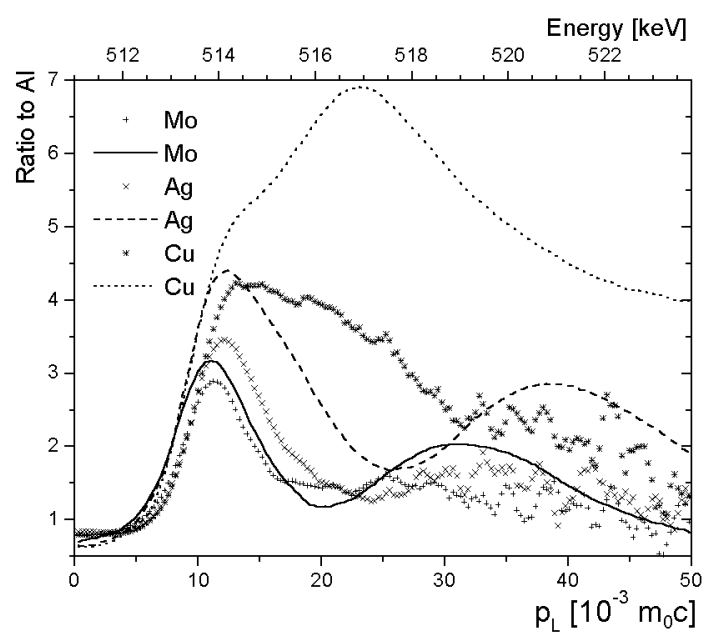

Fig. 11. Comparison between theoretical (from [13]) and experimental ratio curves for (a) $\mathrm{Cu}, \mathrm{Ag}, \mathrm{Au}$, (b) Mo, W. Let us note a normalization to $\mathrm{Al}$, due to lack of literature data for normalization to Si. 
Comparison between our experimental and the theoretical [13] curves is shown for $\mathrm{Mo}, \mathrm{Ag}$, and $\mathrm{Cu}$ in Fig. 11. One notes a good agreement of the general shape. For light elements, like Mo (and also $\mathrm{Fe}, \mathrm{Zn}, \mathrm{Au}, \mathrm{Pb}, \mathrm{W}$, and $\mathrm{Ni}$, not shown for clarity reasons), the agreement between the experiment and the theory is very good also for absolute values of the ratio curves. For 5 th period elements our curves are generally lower than the theory, both in the first and the second peak. A big discrepancy is observed also in absolute values for $\mathrm{Cu}$ [13]; we are not aware of a reason for this.

Some of these discrepancies can be caused by a slightly different energy resolution of the present data $(1.3 \mathrm{keV})$ compared to the resolution (1.5 keV FWHM) assumed in the theoretical analysis. We note that the theory predicts that the overall shape and the position of the ratio peaks do not change much with the detector resolution but the height of these peaks changes in particular if choosing $\mathrm{Al}$ as the reference sample [19].

\section{Semiempirical analysis}

Already in the pioneer work [20] numerous trials of fitting procedures in order to describe the shape of the ratio curves were presented. Myler and Simpson [20] used three overlapping Gaussian curves for approximating the Si-based ratio curves for elements from $Z=27$ to 30 . We show such a trial for present data for atoms from $Z=22$ to 30 in Fig. 12; for space reasons only most significant figures are shown. In $\mathrm{Ti}(Z=22)$ essentially only one Gaussian curve is needed, with only a little addition of a broad peak centered at about $30 m_{0} c$. In $\operatorname{Cr}(Z=24)$ the second Gaussian is needed in order to reproduce the right-hand shoulder of the peak. In Fe, Co, Ni three Gaussians with diminishing amplitudes are visible, see Fig. 12. In $\mathrm{Cu}$, compared to $\mathrm{Ni}$ the amplitude of the first peak (at $13.8 m_{0} c$ ) remains almost the same ( $5.0 \mathrm{in} \mathrm{Ni}$ and $4.8 \mathrm{in} \mathrm{Cu}$, in arbitrary units) but the amplitude of the second peak at about $20 m_{0} c$ rises from 6.4 in $\mathrm{Ni}$ to $8.4 \mathrm{in} \mathrm{Cu}$. Parameters of the three-component Gaussian fit (the peak positions and amplitudes) are compared in Figs. 13a and b.

The weak point of the analysis with three Gaussian components is that, although describing well the curves, it requires as many as 9 fitting parameters. These parameters change with $Z$ with some systematic manner but we cannot attribute to them a significant physical meaning.

As an additional test, we used a simplified method, fitting curves with exponentially-modified Gaussian peak function. These curves require only 4 parameters. As seen from Fig. 14 for $\mathrm{Ti}, \mathrm{V}, \mathrm{Cr}, \mathrm{Fe}, \mathrm{Cu}$, and $\mathrm{Ni}$, the agreement with the overall shape of curves is acceptable for lighter elements, with some discrepancies at higher $p_{\mathrm{L}}$ and is quite good for $\mathrm{Fe}, \mathrm{Ni}$ and $\mathrm{Cu}$. The chi-square values are below 0.01 for all the 9 elements considered. 


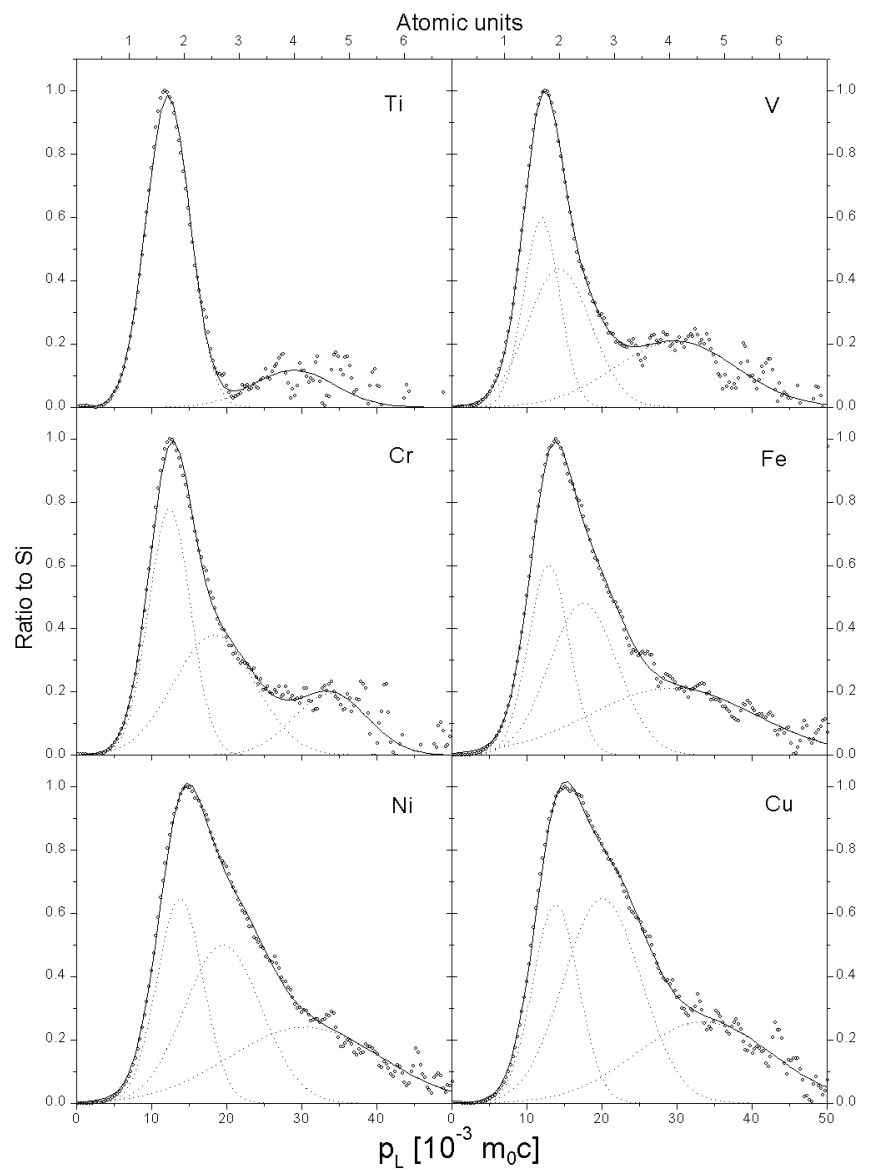

Fig. 12. Fitting of ratio curves with three Gaussians (dotted lines) for selected 4th period elements. Let us note a good agreement of their sums (bold lines) with experimental data. Let us note that amplitudes were normalized to 1.0 .

For 5 th and 6 th period elements this simple fit does not work: addition of a second (non-modified) Gaussian is needed, see Fig. 14 for $\mathrm{W}$ and $\mathrm{Pb}$. In the case of $\mathrm{W}$ this second peak is of almost the same amplitude as the first one; in the case of $\mathrm{Pb}$ the second peak is very weak, amounting to merely $20 \%$ of the first one.

In Fig. 15 we present a plot of the dependence of the peak position vs. $Z$. One notes a simple, linear dependence for $Z=22-30$. In the same figure we compare the peak positions (in atomic units) with the mean values of momenta of $3 d$ electrons from Roothan-Hartree-Fock model [21]. Our data are only somewhat lower than the theoretical values, with the difference rising with the number of $d$ electrons. We note that for spherical orbits the projection $p_{\mathbf{L}}$ of momentum vector $p$ on the observation axis is $p_{\mathrm{L}}=4 p / \pi^{2}=0.4 p$. For $d$ orbitals this projection can be 

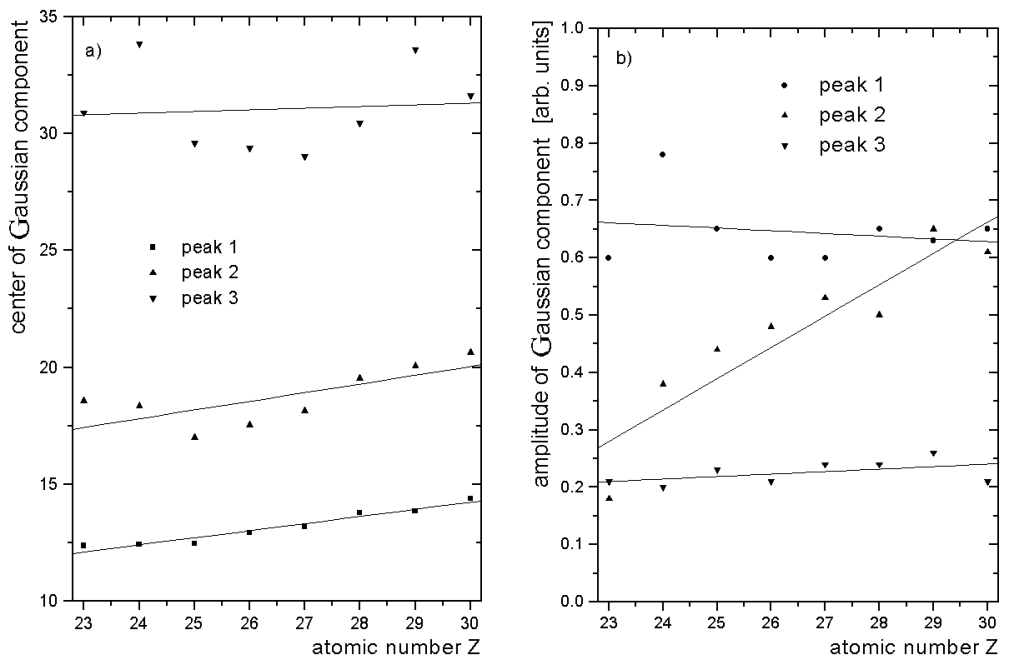

Fig. 13. Parameters of three Gaussian-curves used for fitting 4th period elements spectra, see also Fig. 12. (a) Centroid of Gaussian curves, (b) amplitude of Gaussian peaks.

higher than for spherical orbits, however always lower than $p$. Let us additionally note that the theoretical values reported are for free atoms and do not take into account the orientation of $d$ orbitals in the solid state, influencing our measured values, in particular for monocrystals. Apart from these considerations, the present analysis reproduces qualitatively the general trends predicted by the Hartree-Fock model [21].

Unfortunately, in the subsequent theoretical work [22] for heavy elements $(Z=55-92)$ no values of mean momenta for single orbitals were given. Therefore we can make a comparison only for $\mathrm{Mo}(Z=42)$ and $\mathrm{Ag}(Z=47)$. In the case of these two atoms we can distinguish two peaks (relative figures are not shown). The first one is placed at 1.32 a.u. for Mo and 1.45 a.u. for Ag and broad second one placed at $1.46 \mathrm{a} . \mathrm{u}$. and at $3.71 \mathrm{a} . \mathrm{u}$. for Mo and $\mathrm{Ag}$, respectively. Comparing with calculated values of orbital momenta [21] we can suggest that the first peak originates from $4 d$ (1.92 a.u. for Mo and 2.94 a.u. for $\mathrm{Ag}$ ) orbital and second one from $4 p$ (2.5 a.u.) for Mo and from $5 s$ (6.5 a.u.) for Ag. The lower values measured by us are in agreement with previous considerations.

It seems that in the heavier elements, like $\mathrm{Ag}$ and Au pair, see Fig. 9b, the first peak remains due to annihilation with the outermost orbital ( $4 d$ for $\mathrm{Ag}$ and $5 d$ for $\mathrm{Au}$ ) and the second peak is due to momentum of "inner" $-4 d$ for Au electrons.

This simplified analysis does not take into account momenta of other external orbitals. For Sn we observe only a low peak centered at about 1.8 a.u. and broadened towards higher momenta, see Fig. 7. This peak could correspond to the "outermost" $4 d$ electrons that have the mean momentum of 3.65 a.u. [21]. We note that it agrees qualitatively with the ratio about $1 / 2$ between our mea- 


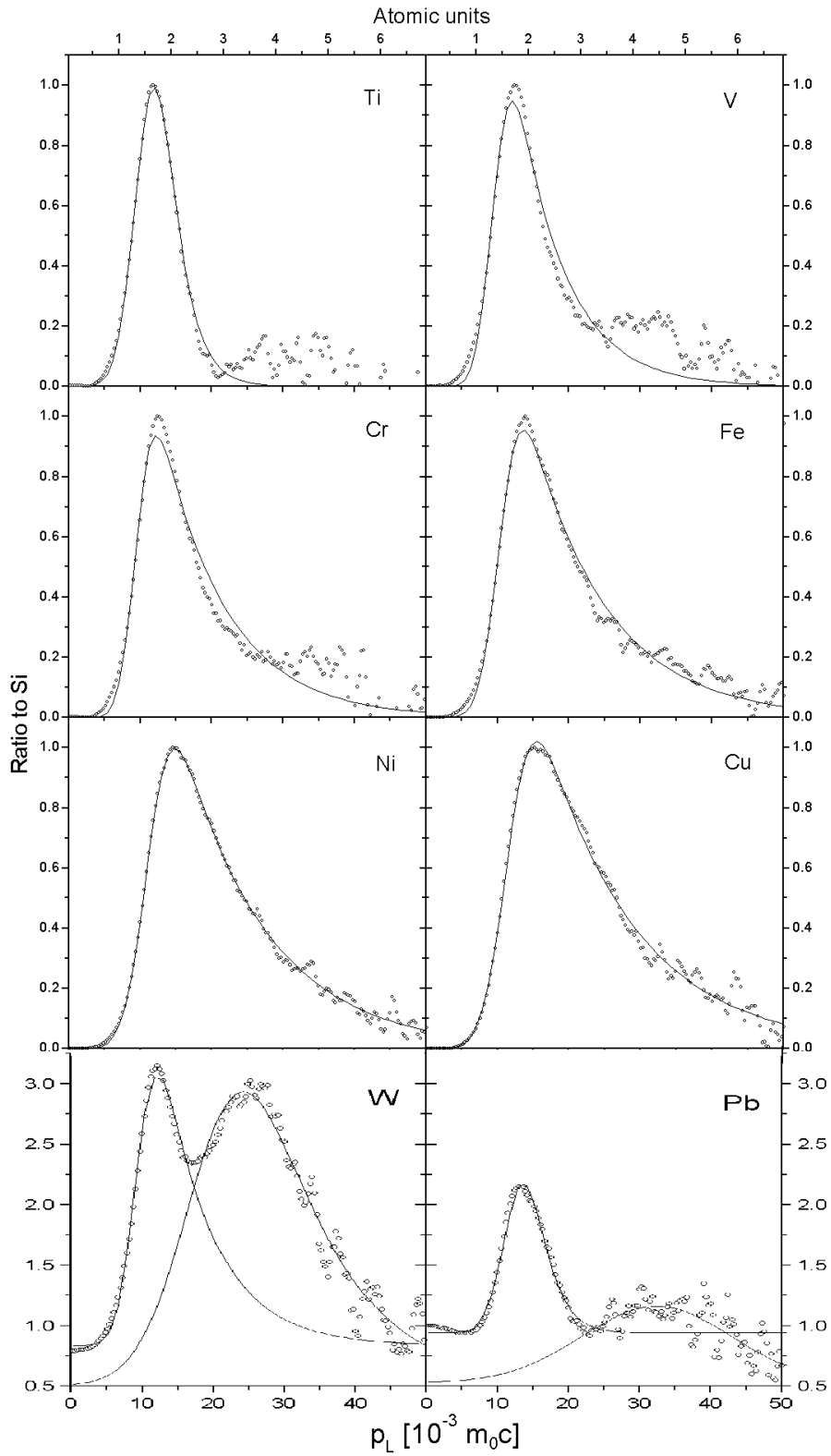

Fig. 14. Fitting of ratio curves with exponentially-modified Gaussian peak function. Let us note that amplitudes for elements from $\mathrm{Ti}$ to $\mathrm{Cu}$ were normalized to 1.0.

sured $p_{\mathrm{L}}$ and the mean theoretical momentum (see Fig. 15). At higher $p_{\mathrm{L}}$ in $\mathrm{Sn}$ we cannot distinguish any clear structure outside our experimental uncertainty. We recall that $5 s$ electrons have the mean momentum of 0.9 a.u., $5 p$ electrons 0.88 a.u. and $3 d$ electrons -10.6 a.u. Let us note that at low $p_{\mathrm{L}}$ the Sn curve exceeds that in $\mathrm{Si}$ (the ratio slightly above 1.0). 


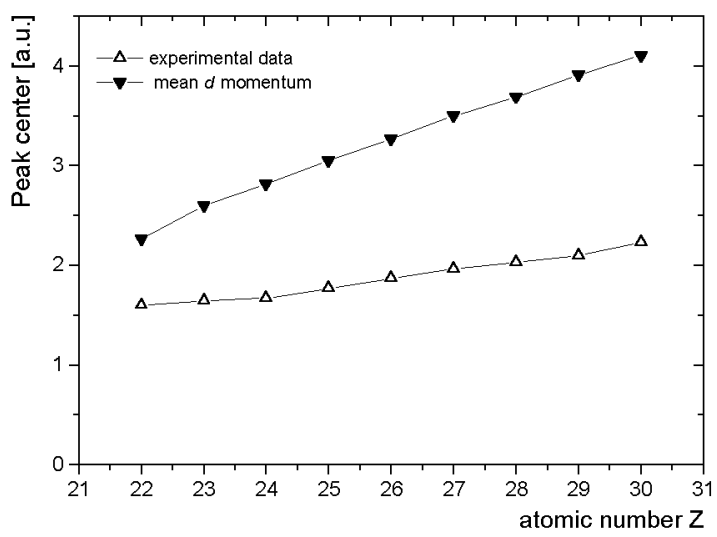

Fig. 15. Position of center of peak exponentially-modified Gaussian function as function of $Z$. Theoretical values, mean values of momentum $p$ from Roothan-Hartree-Fock calculations [21].

\section{Concluding remarks}

The Doppler coincidence proves to be chemically sensitive. In particular, for the 4th period transition-group elements, the ratio curves related to $\mathrm{Si}$ allow to relate the position of peaks to theoretical mean momenta of electrons on the outermost, $d$-orbitals. The position of the first peak seems to be characteristic of the periodic-table group, i.e. for Ag is the same as for Au. Obviously, this simplified analysis shows numerous exceptions.

The heights of the ratio peaks, on the other hand, are very sensitive to defects in samples. As confirmed by lifetime measurements, only defect-free samples show high peaks, close to theoretical [13] values. Examples of Au, Hf, W show that already short annealing times bring the measured lifetimes to the theoretical values. The ratio peaks, on the other hand, continue to rise with further, longer and higher-temperature annealing. As shown by a recent work [10] the Doppler coincidence technique can be also successfully used for alloys.

\section{Acknowledgments}

The work was partially supported by the Ministero della Università e della Ricerca Tecnologica, Cofin 2000 program MM02178957-002.

\section{References}

[1] M. Biasini, Physica B 275, 285 (2000).

[2] M. Hakala, M.J. Puska, R.M. Nieminen, Phys. Rev. B 57, 7621 (1998). 
[3] R.S. Brusa, G.P. Karwasz, N. Tiengo, A. Zecca, F. Corni, R. Tonini, G. Ottaviani, Phys. Rev. B 61, 10154 (2000).

[4] A. Zecca, R.S. Brusa, M.P. Duarte-Naia, G.P. Karwasz, J. Paridaens, A. Piazza, G. Kogel, P. Sperr, D.T. Britton, K. Uhlmann, P. Willutzki, W. Triftshäuser, Europhysics Lett. 29, 617 (1995).

[5] K.G. Lynn, A.N. Goland, Solid State Commun. 18, 1549 (1976).

[6] A. Baranowski, M. Kostrzewa, M. Szuszkiewicz, in: Proc. 33rd Seminar on Positronium Annihilation, Turawa (Poland) 2001, Ed. K. Jerie, University of Opole, Opole 2001, p. 121.

[7] H. Kauppinen, L. Baroux, K. Saarinen, C. Corbel, P. Hautojarvi, J. Phys,, Condens. Matter 9, 5495 (1997).

[8] M. Alatalo, P. Asoka-Kumar, V.J. Ghosh, B. Nielsen, K.G. Lynn, A.C. Kruseman, A. van Veen, T. Korhonen, M.J. Puska, J. Phys. Chem. Solids 59, 55 (1998).

[9] S. Szpala, P. Asoka-Kumar, B. Nielsen, J.P. Peng, S. Hayakawa, K.G. Lynn, H.-J. Gossmann, Phys. Rev. B 54, 4722 (1996).

[10] A. Baranowski, M. Kostrzewa, M. Szuszkiewicz, Acta Phys. Pol. A 99, 329 (2001).

[11] J. De Vries, A. Zecca, R.S. Brusa, R.G. Grisenti, S. Oss, Nucl. Instrum. Methods Phys. Res. A 275, 194 (1989).

[12] P. Asoka-Kumar, M. Alatalo, V.J. Ghosh, A.C. Kruseman, B. Nielsen, K.G. Lynn, Phys. Rev. Lett. 77, 2097 (1996).

[13] V.J. Ghosh, M. Alatalo, P. Asoka-Kumar, B. Nielsen, K.G. Lynn, A.C. Kruseman, P.E. Mijnarends, Phys. Rev. B 61, 10092 (2000).

[14] M. Alatalo, M.J. Puska, Adv. Quant. Chem., in press.

[15] A. Seeger, F. Barnhart, W. Brauer, in: Positron Annihilation, Eds. L. Dorikens-Vanpraet, M. Dorikens, D. Segers, World Sci., Singapore 1989, p. 275.

[16] I.K. McKenzie, in: Positron Solid-State Physics, Eds. W. Brandt, D. Dupasquier, North-Holland, Amsterdam 1983, p. 196.

[17] M.J. Puska, R. Nieminen, Rev. Mod. Phys. 66, 481 (1994).

[18] A. Kluza, W. Rudzińska, W. Szuszkiewicz, Acta Phys. Pol. A 99, 407 (2001).

[19] V.J. Ghosh, B. Nielsen, A. Kruseman, P.E. Mijnarends, A. van Veen, K.G. Lynn, Appl. Surf. Sci. 149, 234 (1999).

[20] U. Myler, P.J. Simpson, Phys. Rev. B 56, 14303 (1997).

[21] J.M. Garcia de la Vega, B. Miguel, At. Data Nucl. Data Tables 54, 1 (1993).

[22] J.M. Garcia de la Vega, B. Miguel, At. Data Nucl. Data Tables 58, 307 (1994). 